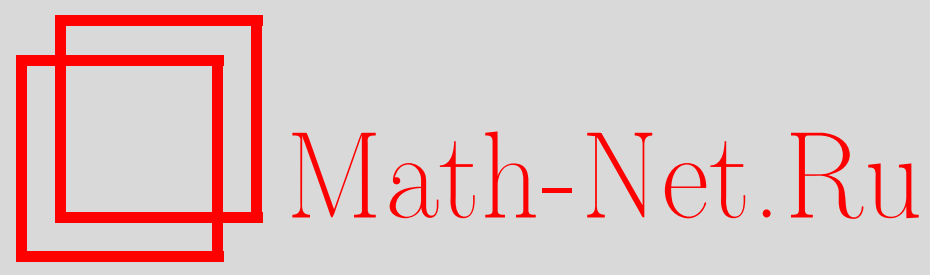

Ю. А. Альпин, Н. А. Корешков, Об одновременной триангулизуемости матриц, Матем. заметки, 2000, том 68, выпуск $5,648-652$

DOI: https://doi.org/10.4213/mzm986

Использование Общероссийского математического портала Math-Net.Ru подразумевает, что вы прочитали и согласны с пользовательским соглашением http://www . mathnet.ru/rus/agreement

Параметры загрузки:

IP : 52.6 .47 .48

26 апреля 2023 г., 14:00:58

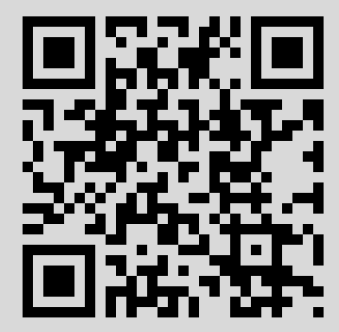




\title{
ОБ ОДНОВРЕМЕННОЙ ТРИАНГУЛИЗУЕМОСТИ МАТРИЦ
}

\author{
Ю. А. Альпин, Н. А. Корешков
}

\begin{abstract}
Установлены два необходимых и достаточных критерия одновременной триангулизуемости двух комплексных матриц. Оба они допускают конечную процедуру проверки. Для доказательства первого критерия привлекаются классические теоремы теории алгебр Ли, причем известные достаточные условия триангулизуемости тоже получают в терминах этой теории естественную интерпретацию. Второй критерий обсуждается в границах ассоциативных алгебр. Решаюшим фактом здесь оказьвается теорема Веддербарна о нильпотентности конечномерной нильалгебры.
\end{abstract}

Библиография: 13 названий.

Введение. Вопрос о том, могут ли две комплексные матрицы быть приведены к верхнему треугольному виду одним подобием - один из старых вопросов линейной алгебры. В принципе он решается следующей теоремой Маккоя [1], [2].

Теорема 1. Матрицы $A$ и В одновременно триангулизуемы тогда и только тогда, когда для всякого многочлена $p(x, y)$ от некоммутируюших переменны $x$ и у матрица $p(A, B)(A B-B A)$ нильпотентна.

Однако, как отмечено в обзоре [3] современного состояния вопроса, доказательство Маккоя сложно и не содержит конечной процедуры проверки выполнения условия теоремы. Более элементарное доказательство Дрейзина, Данги и Грюнберга [4], [5] тоже не дает способа проверки.

В этой заметке доказаны два необходимых и достаточных критерия одновременной триангулизуемости двух матриц. Оба они допускают конечную процедуру проверки. Для доказательства первого критерия привлекаются классические теоремы теории алгебр Ли, причем известные достаточные условия триангулизуемости тоже получают в терминах этой теории естественную интерпретацию. Второй критерий обсуждается в границах ассоциативных алгебр. Решающим фактом здесь оказывается теорема Веддербарна о нильпотентности конечномерной нильалгебры.

1. Триангулизуемость и алгебры Ли. Напомним некоторые определения из теории алгебр Ли. Пусть $G$ - алгебра Ли над полем комплексных чисел. Если $H, T-$ подпространства в $G$, то обозначим через $[H, T]$ подпространство, натянутое на коммутаторы $[h, t], h \in H, t \in T$. Определим индуктивно две серии подпространств:

$$
G^{(k)}=\left[G^{(k-1)}, G^{(k-1)}\right], \quad G_{k}=\left[G_{k-1}, G\right], \quad G^{(0)}=G_{0}=G .
$$

648 (C) Ю.А. Альпин, Н.А. Корешков 2000 
Алгебра $G$ называется разрешимой, если

$$
G \supset G^{(1)} \supset \cdots \supset G^{(r)}=\{0\}
$$

и нильпотентной, если

$$
G \supset G_{1} \supset \cdots \supset G_{s}=\{0\} .
$$

Поскольку $G^{(k)} \subseteq G_{k}$, то нильпотентность алгебры $G$ влечет ее разрешимость. На самом деле - заметим это для дальнейшего - для разрешимости $G$ достаточно, чтобы коммутант $G_{1}=[G, G]$ был нильпотентной алгеброй Ли.

Теория алгебр Ли содержит следующий ответ на вопрос о совместной триангулизуемости матриц. Теорема Ли утверждает, что алгебра Ли линейных операторов в конечномерном линейном пространстве разрешима тогда и только тогда, когда существует базис этого пространства, в котором матрицы всех операторов алгебры имеют верхний треугольный вид (см., например, [6]). Таким образом, матрицы $A, B$ одновременно триангулизуемы в точности тогда, когда порожденная ими алгебра Ли $L=L(A, B)$ разрешима.

Покажем, что теорема Маккоя может быть получена как несложное следствие теоремы Ли.

ТЕорема 2. Пусть $A, B$ - комплексные матрицы порядка $n \geqslant 2$. Следующие утверждения равносильны:

1) матрицы $A$ и В одновременно триангулизуемы,

2) для всякого многочлена $p(x, y)$ от некоммутируюших переменных $x, y$ матрииа $p(A, B)(A B-B A)$ нильпотентна,

3) алгебра Ли $L=L(A, B)$ разрешима,

4) коммутант $L_{1}=[L, L]$ нильпотентен как подалгебра $L$.

ДокаЗАТЕльство. Импликация 1) $\Longrightarrow 2$ ) очевидна. Докажем, что 2) влечет 4). Для этого заметим, что любая матрица $p(A, B)(A B-B A) q(A, B)$, где $p(x, y), q(x, y)$ многочлены, ассоциативно нильпотентна, поскольку нильпотентна матрица $q(A, B) \times$ $p(A, B)(A B-B A)$. Линейная оболочка $H$ элементов $p(A, B)(A B-B A) q(A, B)$ является алгеброй Ли, имеющей базис из нильпотентньх элементов. По теореме Энгеля [7] такая алгебра есть нильпотентная алгебра Ли. Поскольку $[L, L] \subseteq H$, то и $[L, L]$ нильпотентная алгебра. Таким образом, условие 4) выполняется. Импликация 4) $\Longrightarrow ~ 3)$ справедлива ввиду замечания, сделанного в начале пункта. Наконец, из 3) вытекает 1) по теореме Ли. Теорема доказана.

Заметим, что теорема Ли, как и теорема Маккоя, не дает ответа на вопрос, как за конечное число шагов проверить, триангулизуемы ли две матрицы одновременно. По-видимому, классики не интересовались этим вопросом. В конще пункта мы приведем способ проверки, но прежде рассмотрим с точки зрения теории алгебр Ли два известных достаточных условия триангулизуемости.

Если матрицы $A$ и $B$ перестановочны, то их одновременная триангулизуемость - известньй факт линейной алгебры [8, задача 6.3.44;], [2]. Коммутант соответствующей алгебры Ли в этом случае тривиален: $L_{1}=\{0\}$. Говорят, что матрицы $A, B$ квазиперестановочны, если

$$
[A, B] \neq 0, \quad[[A B-B A], A]=[[A B-B A], B]=0 .
$$


Теорема 3 (малая теорема Маккоя [9], [3]). Квазиперестановочные матрииы $A$ $u$ м могут быть приведены $к$ верхнему треугольному виду одним и тем же подобием.

ДокАЗАТЕЛЬСТво. Условие теоремы иньми словами означает, что $L_{1} \neq\{0\}$, но $L_{2}=\{0\}$, причем матрищы $A, B,[A, B]$ линейно независимы. Таким образом, в этом случае $L=L(A, B)$ - трехмерная нильпотентная (и, следовательно, разрешимая) алгебра Ли. Применение теоремы Ли завершает доказательство.

Дрейзин [10], [3] предложил следующее обобщение свойства перестановочности. Положим $C_{0}=\{A, B\}$ и определим индуктивно множества

$$
C_{k}=\left\{[A, X], X \in C_{k-1}\right\} \cup\left\{[B, X], X \in C_{k-1}\right\} .
$$

Если для некоторого $k C_{k}=\{0\}$, то говорят, что матрицы $A$ и $B$ обладают свойством обобщенной квазиперестановочности. Очевидно, если $C_{k}=\{0\}$, то $C_{k+1}=$ $C_{k+2}=\cdots=\{0\}$.

Теорема 4 (Дрейзин). Если матрииы $A$ и В обладают свойством обобщенной квазиперестановочности, то они могут быть приведены к верхнему треугольному виду одним подобием.

ДоКАЗАТЕЛЬСтво. Любой элемент алгебры $L=L(A, B)$ с помощью тождества Якоби и свойства антикоммутативности преобразуется в линейную комбинацию правонормированных мономов вида

$$
\left[X_{1},\left[X_{2}, \ldots,\left[X_{k}, X_{k+1}\right], \ldots\right]\right], \quad X_{i} \in\{A, B\}, \quad i=1, \ldots, k+1
$$

Всевозможные такие коммутаторы для фиксированного $k$ составляют $C_{k}$. Нетрудно заметить следующую связь между коммутантами $L_{k}$ и множествами $C_{k}$ (угловые скобки означают взятие линейной оболочки):

$$
\forall k \geqslant 0 \quad C_{k} \subset L_{k}, \quad L_{k} \subseteq\left\langle C_{k} \cup C_{k+1} \cup \cdots\right\rangle
$$

Отсюда видно, что $C_{k}=\{0\} \quad \Longleftrightarrow \quad L_{k}=\{0\}$ и, стало быть, обобщенная квазиперестановочность матрищ $A, B$ и нильпотентность порожденной ими алгебры Ли - эквивалентные свойства. Применяя, как и в предыдущем случае, теорему Ли, получаем утверждение теоремы Дрейзина.

Чтобы получить конструктивную форму критерия триангулизуемости, используем условие Картана разрешимости алгебры Ли. Это условие гласит [11]: если след ассоциативного произведения любых двух элементов алгебры Ли линейных операторов конечномерного векторного пространства равен нулю, то алгебра разрешима.

Теорема 5. Матрииы $A$ и $B$ порядка п могут быть приведены к верхнему треугольному виду одним и тем же подобием тогда и только тогда, когда равен нулю след ассочиативного произведения любых двух мономов вида

$$
\left[X_{1},\left[X_{2}, \ldots,\left[X_{k}, X_{k+1}\right] \ldots\right]\right], \quad X_{i} \in\{A, B\}, \quad k \leqslant n^{2}-1 .
$$


ДоКАЗАТЕЛЬСтво. В части необходимости утверждение теоремы очевидно. Пусть теперь вьполнено условие теоремы. Уточняя применительно к $L_{1}=[L, L]$ связь между $L_{k}$ и $C_{k}$, сформулированную в доказательстве теоремы 3 , отметим, что $L_{1}=\left\langle C_{1} \cup\right.$ $\left.C_{2} \cup \cdots \cup C_{m}\right\rangle$, где $m$ - наименьшее число, для которого $\left\langle\mathrm{C}_{0} \cup C_{1} \cup \cdots \cup C_{m}\right\rangle=\left\langle C_{0} \cup C_{1} \cup \cdots\right.$ $\left.\cup C_{m} \cup C_{m+1}\right\rangle$. Очевидно, $m \leqslant n^{2}-1$. Нам дано, что условие Картана вьполнено для любых элементов множества, натягивающего $L_{1}$ как линейное пространство. Но тогда оно выполнено в силу линейности функции следа для любых элементов $L_{1}$. Следовательно, $L_{1}$ - разрешимая алгебра. Но тогда и $L$ - разрешимая алгебра, к которой применима теорема Ли. Теорема доказана.

2. Триангулизуемость и теорема Веддербарна. В этой части все алгебры ассоциативные. Обозначим $\mathscr{A}(A, B)$ подалгебру ассоциативной алгебры $M_{n}(\mathbb{C})$ комплексных матрищ порядка $n$, порожденную матрищами $A$ и $B$. Условие Маккоя в стандартных для теории алгебр терминах пересказывается так: левьй идеал $I=\mathscr{A}(A, B) \times$ $(A B-B A)$, порожденньй матрицей $A B-B A$, должен быть нильидеалом. Желая выделить содержательный случай, считаем далее, что $A B-B A \neq 0$. Согласно теореме Веддербарна [12] нильидеал конечномерной алгебры является нильпотентньм идеалом. Следовательно, если условие Маккоя выполнено, то имеем убывающую цеп

$$
I \supset I^{2} \supset \cdots \supset I^{s}=\{0\} .
$$

После этого замечания доказательство теоремы Маккоя становится совсем простым. Из (1) вытекает, что вложение

$$
\mathbb{C}^{n} \supseteq I\left(\mathbb{C}^{n}\right)
$$

является на самом деле строгим включением, ибо в предположении равенства получаем $\mathbb{C}^{n}=I^{k}\left(\mathbb{C}^{n}\right), k=1,2, \ldots$ Но это противоречит нильпотентности $I$. Подпространство $I\left(\mathbb{C}^{n}\right)$ инвариантно относительно операторов $A$ и $B$; следовательно, в подходящем базисе матрицы приобретут вид

$$
\left(\begin{array}{cc}
A_{1} & G \\
0 & A_{2}
\end{array}\right), \quad\left(\begin{array}{cc}
B_{1} & H \\
0 & B_{2}
\end{array}\right),
$$

где $A_{1}, B_{1}$ - матрицы сужений операторов $A, B$, действуюших в $I\left(\mathbb{C}^{n}\right)$, а $A_{2}, B_{2}$ - матрицы фактор-операторов, действующих в фактор-пространстве $\mathbb{C}^{n} / I\left(\mathbb{C}^{n}\right)$. Аналогичньй смысл имеют диагональные блоки для любой из матрищ алгебры, получившихся после перехода к новому базису. Поскольку образ оператора $A B-B A$ лежит в $I\left(\mathbb{C}^{n}\right)$, то его фактор-оператор есть нулевой оператор. Таким образом, $A_{2} B_{2}-B_{2} A_{2}=0$, матрицы $A_{2}, B_{2}$ перестановочны и, следовательно, совместно триангулизуемы. Для матриц $A_{1}, B_{1}$ порядка меньше, чем $n$, выполнено условие Маккоя и к ним можно применить предыдущие рассуждения. Продолжая редукцию по порядку, придем к доказательству теоремы.

Чтобы получить конструктивную форму теоремы Маккоя, используем простое следствие теоремы Веддербарна [13]. Для того чтобы подалгебра алгебры $M_{n}(\mathbb{C})$ была нильпотентна, необходимо и достаточно, чтобы существовал ее базис, составленный из матриц с нулевым следом.

Идеал $I=\mathscr{A} A(A, B)$ - подалгебра алгебры $M_{n}(\mathbb{C})$. Ее базис заведомо можно выбрать из конечного множества матриц

$$
\left\{A_{1} \ldots A_{k}(A B-B A), A_{i} \in\{A, B\}, i=1, \ldots, k, 0 \leqslant k \leqslant n^{2}-1\right\} .
$$


Теорема 6. Матрицы $A$ и В одновременно триангулизуемы тогда и только тогда, когда след любой матричы из конечного множества (2) равен нулю.

\section{СПИСОК ЦИТИРОВАННОЙ ЛИТЕРАТУРЫ}

[1] McCoy N.H. On the characteristic roots of matrix polynomials // Bull. Amer. Math. Soc. 1936. V. 42. P. 592-600.

[2] Хорн Р., Джонсон Ч. Матричный анализ. М.: Мир, 1989.

[3] Икрамов Х. Д., Савельева Н.В., Чугунов В.Н. О рациональных критериях существования общих собственных векторов или инвариантных подпространств // Программирование. 1997. № 3 . С. 43-57.

[4] Drazin M.P., Dungey J. W., Gruenberg K. W. Some theorems on commutative matrices // J. London Math. Soc. 1951. V. 26. P. 221-228.

[5] Прасолов В. В. Задачи и теоремы линейной алгебры. М.: Наука, 1996.

[6] Бахтурин Ю. А. Основные структуры современной алгебры. М.: Наука, 1990.

[7] Капланский И. Алгебры Ли и локально компактные группы. М.: Мир, 1974.

[8] Икрамов Х. Д. Задачник по линейной алгебре. М.: Наука, 1975.

[9] McCoy N.H. On quasi-commutative matrices // Trans. Amer. Math. Soc. 1934. V. 36. P. 327-340.

[10] Drazin M.P. Some generalizations of matrix commutativity // Proc. London Math. Soc. 1951. V. 1. P. 222-231.

[11] Постников М. М. Группы и алгебры Ли. М.: Наука, 1982.

[12] Пирс Р. Ассоциативные алгебры. М.: Мир, 1986.

[13] Чеботарев Н. Г. Введение в теорию алгебр. М.: Гостехиздат, 1949.

Казанский государственный университет 